\title{
In silico identification of expressed sequence tags based simple sequence repeats (EST-SSRs) markers in Trifolium species
}

\author{
Siddra Ijaz ${ }^{\mathrm{a}, *}$, Imran Ul Haq ${ }^{\mathrm{b}}$, Bukhtawer Nasir ${ }^{\mathrm{a}}$ \\ a Centre of Agricultural Biochemistry and Biotechnology (CABB), University of Agriculture, University \\ Road, Faisalabad, Pakistan \\ b Department of Plant Pathology, University of Agriculture, University Road, Faisalabad, Pakistan.
}

*Corresponding author, e-mail: siddraijazkhan@yahoo.com

Received 5 May 2019

Accepted 6 Dec 2019

\begin{abstract}
The EST-SSRs (genic SSRs) are the microsatellites in expressed sequence tags (ESTs). Advancement in functional genomics has contributed mainly for the identification of ESTs in different species and made them available in databases. They are of transcribe regions, so are more conserved and considered with more cross transferability across the taxonomic borders. In Trifolium alexandrinum (Berseem), no expressed sequence tags (ESTs) are available in NCBI database yet. Therefore, in this study by considering the cross transferability potential of EST-SSRs, expressed sequence tags of Trifolium pratense and Trifolium repens were retrieved from NCBI database and their EST-SSRs were in silico identified. We retrieved 1014 ESTs for SSR identification from both Trifolium species and identified 198 EST-SSRs from them. In identified EST-SSRs, trinucleotide SSRs repeats were found to be more frequent. Of these 198 in silico identified EST-SSRs, 44 showed cross species amplification in T. alexandrinum and was proved for their transferability potential. The identified EST-SSRs could be a great source for genetic diversity and population genetics studies of Trifolium species. These EST-SSRs could be further screened to investigate their linkage to disease resistance in Trifolium species.
\end{abstract}

KEYWORDS: expressed sequence tags, simple sequence repeats, microsatellite, berseem, Trifolium species

\section{INTRODUCTION}

Leguminous crops have global importance towards the sustainable agriculture. These crops are rich in protein and oil contents that make them good nutritional source for the members of kingdom Animalia [1]. Nitrogen fixation is one of the remarkable properties of their candidate species that is being used to enhance soil fertility. However, the important constituents of these species are their natural composite or secondary metabolites that involve in symbiosis as well as in plant stress responses $[2,3]$. Apart from all, their recognition is also existed as major intercropping species. In this respect, clover (Trifolium spp. L) is distinguishable group of legume crops that belongs to family Fabaceae and subfamily Faboideae. Whereas this genus; Trifolium consisted of mainly 250 annual or perennial species, native to Middle East, Europe, America and Africa [4]. Of them, twenty species $(10 \%)$ are important to feed animals and are well known forage crops of several regions [5]. Normally these species are grown with companion grasses like rangeland but their silages also have many uses.

The most important species of Trifolium genus are T. repens (white clover), T. pratense (red clover) and T. alexandrinum (Egyptian clover). Among them, T. alexandrinum is of great importance in terms of animal feed [6]. It has been widely cultivated as multi-cut annual grazing crop in Africa and Asia, particularly in Indo-Pak [7]. The good quality, high yielding fodder crops with high digestibility in livestock are valuable features of berseem crop [8]. Moreover, the genetic analysis and characterization of this crop for diversity and for marker-assisted breeding is an important and preliminary step toward crop improvement [9].

Simple-sequence repeats (SSRs) markers are gaining importance due to their high polymorphic rate, high reproducibility, multi-allelic and codominant nature [10]. These features impart distinctness to SSR markers for exploring genomic variation and thereby make them valued for diversity analysis and marker assisted selection [11]. SSRs are abun- 
dant, frequently occur in eukaryotic genome, and widely used in variation study of several legume crops including, white clover, peanut, pigeon pea, mung bean and berseem clover [12]. However, de novo development of genomic SSR is problematic in terms of cost, time and species specific property thus makes them not readily transferable to the other species. Therefore, the possible solution is to identify the genic SSRs or EST-SSRs to crosstaxonomic boundaries [13].

Expressed sequence tags (ESTs) are a short sequence of cDNA (complementary DNA) that give more feasibility and specificity of results towards high quality nuclear marker development. The cross transferability of EST-SSRs has been identified and evaluated in several legume crops including pea, fababean and chickpea, mungbean and dolichos bean [14]. Hence, in this research study, we have tried to identify the SRRs from EST sequences of T. repens and T. pratense through bioinformatic tools, which may have cross transferability into T. alexandrinum (Berseem) and thereby could be putative EST-SSR markers for molecular characterization.

\section{METHODS}

\section{Mining of ESTs sequences}

A total of 1014 ESTs sequences of T. pratense and T. repens were retrieved from the NCBI (National Center for Biotechnology Information) database. The non-redundancy of these ESTs were analyzed using SEQMAN PRO v. 7.1.0.

\section{EST-SSRs detection}

The FASTA formatted file were uploaded in WEBSAT program (microsatellite identification software tool). The criteria set for the detection of SSRs from ESTs sequences were 6 repeat units for di-, 4 for tri-, and 3 for tetra-, penta- and hexanucleotides.

\section{Primer designing for SSR markers}

For SSR markers, primers were designed from the flanking region of identified SSRs. Primer designing and analyzing tools provided by Integrated DNA Technology (IDT) services (eu.idtdna.com/pages) were used to design EST-SSR based primer pairs.

\section{DNA extraction and PCR analysis}

Three cultivars of T. alexandrinum (berseem), Agaite, Pachate and Anmol, were used to assess the cross transferability of identified EST-SSR. Total genomic DNA of these cultivars was extracted using modified CTAB method [15], and maintained in
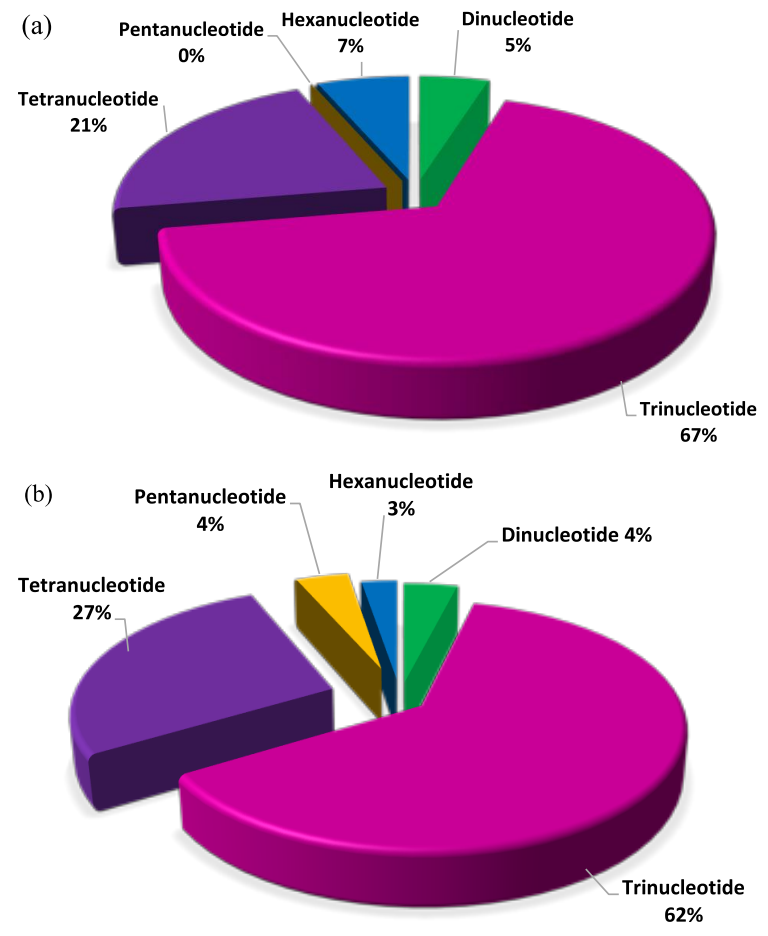

Fig. 1 Distribution frequency of dinucleotide, trinucleotide, tetranucleotide, pentanucleotide and hexanucleotide EST-SSRs in ESTs of (a) T. repens and (b) T. pratense.

TE buffer. The quantification was performed by UV visible NANODROP (8000 Spectrophotometer, Thermo Scientific). PCR were carried out in 96 well thermal cycler (peqSTAR) with $50 \mu 1$ of reaction mixture comprising of High-Fidelity PCR Master Mix (Thermo Scientific). The amplified PCR products were resolved on $2 \%$ high resolution Agarose gel and were visualized under UV light using Gel Documentation system (GDS) of BioRad, USA.

\section{RESULTS AND DISCUSSION}

Molecular markers are the promising tools to measure genetic divergence in several plant species including isozyme, AFLP (amplified fragment length polymorphism), RAPD (random amplified polymorphic DNA), ISSR (inter simple sequence repeats) and SSR (simple sequence repeats) [16-18]. Hence, in this study, 198 SSRs were identified from 1014 ESTs sequences of T. pratense and T. repens (Table S1). Of these total identified SSRs, 121 EST-SSRs were identified from ESTs sequences of T. pratense, whereas 77 EST-SSRs were identified from ESTs sequences of $T$. repens. Trinucleotide 


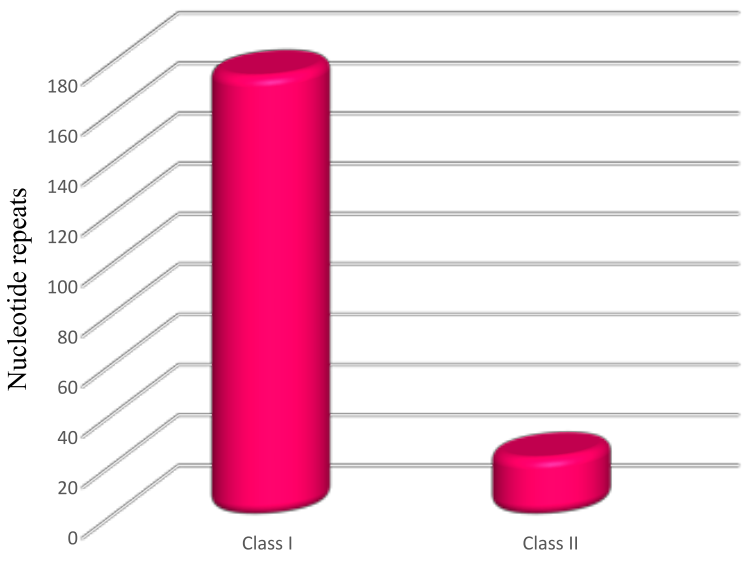

Fig. 2 Classes of EST-SSRs based on nucleotide repeats.

was observed to be more frequent type of repeat in both Trifolium species. In case of T. repens, 82 trinucleotide $(67 \%), 25$ tetranucleotide $(21 \%)$, 8 hexanucleotide (7\%), 6 dinucleotide (5\%) and zero pentanucleotide SSRs (0\%) were observed (Fig. 1a). However, in T. pratense, 48 trinucleotide (62\%), 21 tetranucleotide (27\%), 3 dinucleotide (4\%), 3 pentanucleotide (4\%) and 2 hexanucleotide SSRs (3\%) were observed (Fig. 1b). The nucleotide repeats of identified EST-SSR were grouped into two classes; Class I (12-20 nt) and Class II ( $\geqslant 20 \mathrm{nt})$. The Class I repeats were found to be more frequent (Fig. 2).

A total of 104 different types of EST-SSRs motifs were identified which belong to 5 different types of nucleotide repeats, 8 different types of dinucleotide repeats, 44 different types of trinucleotide repeats, 35 different types of tetranucleotide repeat, 3 different types of pentanucleotide repeats and 9 different types of hexanucleotide repeats. The repeat type CT/AG (4) was more abundant in dinucleotide EST-SSRs, followed by TC/GA (2), AT/TA (1), GA/TC (1) and CA/TG (1) (Fig. 3a). However, repeat type GAA/TTC (10) was abundant in trinucleotide repeats followed by CAA/TTG (7), TGG/CCA (7), CAC/GTG (6), GAT/ATC (6), ACC/GGT (6), ATC/GAT (6), TTC/GAA (6), TCA/TGA (5), TCT/AGA (5), ATT/AAT (4), AAG/CTT (4), ACA/TGT (3), AGG/CCT (3), CTT/AAG (3), GGT/ACC (3), CCG/CGG (3), TGT/ACA (3), TAA/TTA (2), AAT/ATT (2), ATA/TAT (2), AAC/GTT (2), CCT/AGG (2), CTC/GAG (2), CCA/TGG (2), TGC/GCA (2), GTA/TAC (2), CGC/GCG (2), CAT/ATG (2), CAG/CTG (2), TAC/GTA (2), GCT/AGC (2), GCC/GGC (1), CTG/CAG (1),
Table 1 EST-SSRs primers that showed $(\checkmark)$ and did not show $(X)$ cross species amplification in T. alexandrinum in PCR analysis.

\begin{tabular}{|c|c|c|c|c|c|c|c|}
\hline \multicolumn{2}{|c|}{ Primer Agaite } & \multicolumn{2}{|c|}{ Pachate Anmol } & \multicolumn{2}{|c|}{ Primer Agaite } & \multicolumn{2}{|c|}{ Pachate Anmol } \\
\hline TP5 & $\checkmark$ & $\checkmark$ & $\checkmark$ & TR11 & $\checkmark$ & $x$ & $\checkmark$ \\
\hline TP8 & $\checkmark$ & $\checkmark$ & $\checkmark$ & TR17 & $\checkmark$ & $x$ & $\times$ \\
\hline TP10 & $\checkmark$ & $\checkmark$ & $\times$ & TR19 & $\checkmark$ & $\checkmark$ & $\checkmark$ \\
\hline TP11 & $\checkmark$ & $x$ & $\times$ & TR28 & $\checkmark$ & $\checkmark$ & $\checkmark$ \\
\hline TP14 & $\checkmark$ & $\checkmark$ & $\checkmark$ & TR29 & $x$ & $\checkmark$ & $\checkmark$ \\
\hline TP21 & $\times$ & $\times$ & $\checkmark$ & TR31 & $\checkmark$ & $x$ & $\times$ \\
\hline TP22 & $x$ & $\checkmark$ & $\times$ & TR35 & $\checkmark$ & $\checkmark$ & $\checkmark$ \\
\hline TP23 & $\checkmark$ & $\checkmark$ & $\checkmark$ & TR37 & $\checkmark$ & $\checkmark$ & $\checkmark$ \\
\hline TP29 & $\checkmark$ & $\checkmark$ & $\checkmark$ & TR45 & $\times$ & $\checkmark$ & $\checkmark$ \\
\hline TP37 & $x$ & $\times$ & $\checkmark$ & TR48 & $x$ & $x$ & $\checkmark$ \\
\hline TP45 & $x$ & $\checkmark$ & $\checkmark$ & TR54 & $\checkmark$ & $x$ & $x$ \\
\hline TP48 & $x$ & $\checkmark$ & $\times$ & TR55 & $x$ & $\checkmark$ & $x$ \\
\hline TP50 & $\checkmark$ & $\checkmark$ & $\checkmark$ & TR57 & $\checkmark$ & $\checkmark$ & $x$ \\
\hline TP51 & $\checkmark$ & $\checkmark$ & $\checkmark$ & TR71 & $x$ & $\checkmark$ & $\checkmark$ \\
\hline TP55 & $\checkmark$ & $\checkmark$ & $\checkmark$ & TR75 & $\checkmark$ & $\checkmark$ & $\checkmark$ \\
\hline TP59 & $\checkmark$ & $\checkmark$ & $\checkmark$ & TR84 & $\checkmark$ & $\checkmark$ & $\checkmark$ \\
\hline TP63 & $\checkmark$ & $x$ & $\times$ & TR86 & $\checkmark$ & $\checkmark$ & $\checkmark$ \\
\hline TP65 & $x$ & $\checkmark$ & $\checkmark$ & TR94 & $x$ & $\checkmark$ & $\times$ \\
\hline TP72 & $x$ & $\checkmark$ & $\times$ & TR109 & $x$ & $\checkmark$ & $\checkmark$ \\
\hline TR6 & $\checkmark$ & $\checkmark$ & $\checkmark$ & TR114 & $\checkmark$ & $x$ & $\times$ \\
\hline TR9 & $x$ & $x$ & $\checkmark$ & TR115 & $\checkmark$ & $\checkmark$ & $\checkmark$ \\
\hline TR10 & $\checkmark$ & $\checkmark$ & $\checkmark$ & TR120 & $\checkmark$ & $\checkmark$ & $\checkmark$ \\
\hline
\end{tabular}

AGA/TCT (1), TCG/CGA (1), GTT/AAC (1), TTA/TAA (1), GAG/CTC (1), GGA/GCC (1), TGA/TCA (1), GCG/CGC (1), GTA/CAT (1), and ACT/AGT (1) (Fig. 3b). In tetranucleotide repeats, repeat type TTCC/GGAA (3) was frequently present followed TTAT/ATAA (2), ATTT/AAAT (2), TTTC/GAAA (2), TGTT/AACA (2), TCTT/AAGA (2), CCAA/TTGG (2), CATA/TATG (2), AATA/TATT (2), CTTC/GAAG (2), AAAC/GTTT (1), GAAA/TTTC (1), ACCT/AGGT (1), TAGA/TCTA (1), TAGT/ACTA (1), CTAG/CTAG (1), TTAA/TTAA (1), TGAT/ATCA (1), CCAT/ATGG (1), TCAC/GTGA (1), CTTT/AAAG (1), TAAA/TTTA (1), TAAT/ATTA (1), TCGC/GCGA (1), TTTG/CAAA (1), ATTC/GAAT (1), TTCA/TGAA (1), TCTA/TAGA (1), CAAT/ATTG (1), ATTA/TAAT (1), AATC/GATT (1), GCAT/CGTA (1), ATCA/TGAT (1), ACTA/TAGT (1), and AGTG/CACT (1) (Fig. 3c). In case of pentanucleotide repeats, no type was found frequent and all pentanucleotide repeat types were appeared singly as GAAAA/TTTTC (1), CAAAC/GTTTG (1), and GAATC/GATTC (1) (Fig. 3d). In hexanucleotide repeats, the repeat type CCAAAC/GTTTGG (2) was frequent followed by GATTTT/AAAATC (1), CCATCA/TGATGG (1), TTCTCT/AGAGAA (1), TTTGAT/ATCAAA (1), ACCTCC/GGAGGT (1), TGCACC/GGTGCA (1), TGATGG/CCATCA (1), and GTTGGT/ACCAAC (1) (Fig. 3e).

We identified 198 SSRs from 1014 sequences (121 from T. pratense, 77 from T. repens) that added 


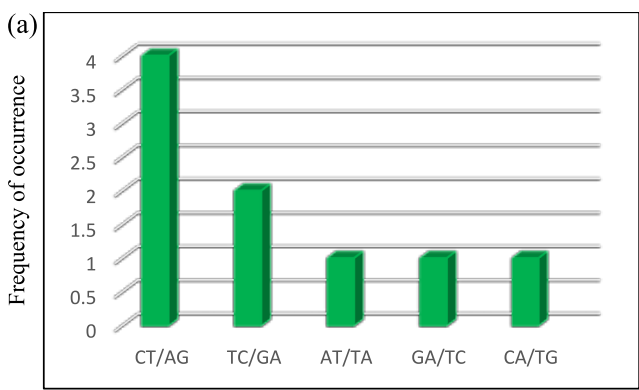

(c)

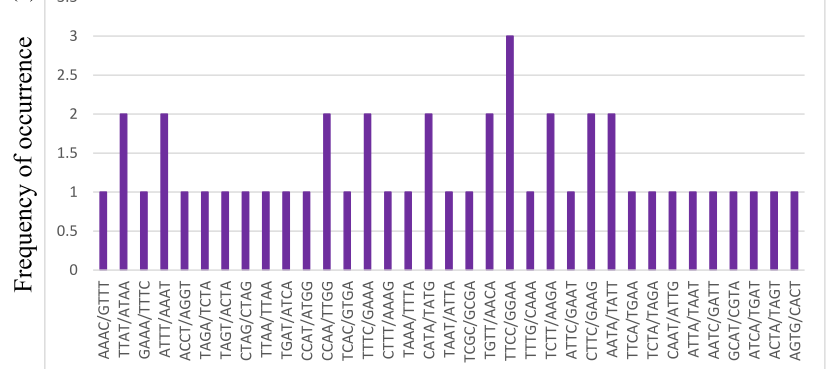

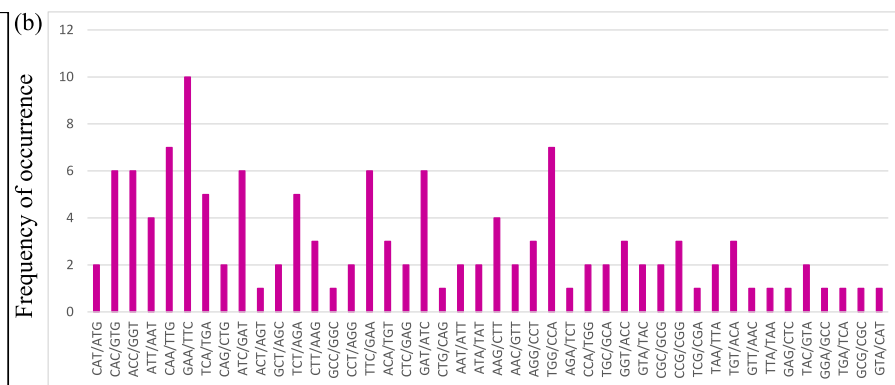

(d)

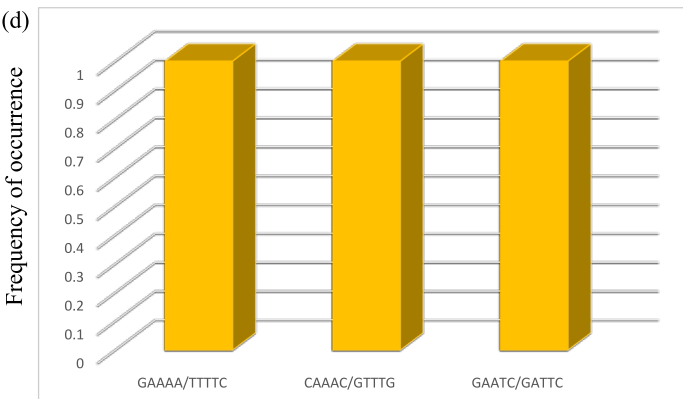

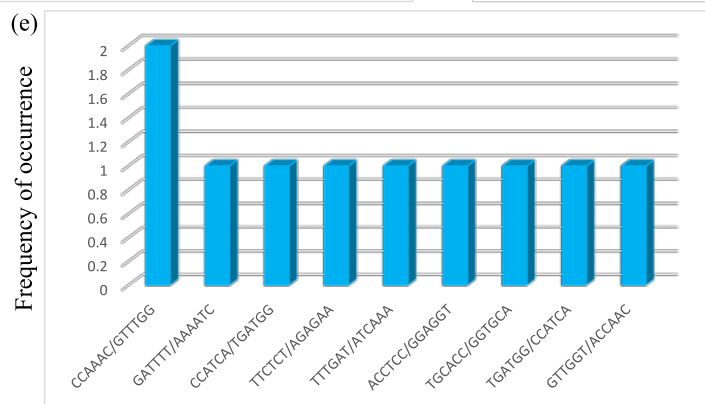

Fig. 3 Frequency of occurrence of different types of EST-SSRs motifs comprising of (a) dinucleotide, (b) trinucleotide, (c) tetranucleotide, (d) pentanucleotide, and (e) hexanucleotide in ESTs sequences.

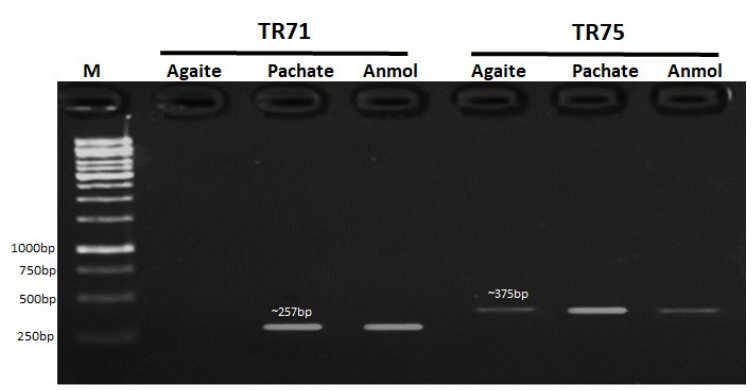

Fig. 4 EST-SSRs primers based PCR amplification in T. alexandrinum. $\mathrm{M}=1 \mathrm{~Kb}$ DNA ladder; TR71 and TR75 = EST-SSRs specific primers; Agaite, Pachate, Anmol = cultivars of T. alexandrinum.

confirmation towards presence of SSR in EST sequence as identified in mungbean. The SSR development through EST database has become an efficient choice with time saving and low cost option for germplasm characterization, comparative genome mapping and linkage analysis [19]. Moreover, the identified genic SSRs from Trifolium spp. were able to be applied for their cross transferability with berseem (T. alexandrinum). This would be helpful to study different aspects of its genomics in a more precise way, which was still missing due to the unavailability of ESTs in T. alexandrinum. From the PCR analysis of these 198 EST-SSRs based primers, 44 primers showed cross-species amplification in T. alexandrinum. PCR pattern of some representative markers is given in Fig. 4. Of these 44 primers, 25 primers were from EST-SSRs of T. repens and 19 were from T. pratense (Table 1).

\section{CONCLUSION}

Simple sequence repeats are the molecular markers of choice for genetic diversity studies due to their highly specific nature and reproducibility. As the unavailability of genetic information record, functional 
genomic studies are very limited in T. alexandrinum. The inaccessibility of genic SSRs for diversity analysis and population genetics of T. alexandrinum in more meaningful way has been becoming the major hurdle. Because in this species, expressed sequence tags are not available yet. Hence, this study would be an imperative contribution for population genetics studies in T. alexandrinum. As the ESTs of its closely related species, $T$. pratense and $T$. repens are available. Therefore, in this study, their ESTs were retrieved, subjected to in silico characterization to find EST-SSRs and were then analyzed for their cross species transferability in T. alexandrinum. In 198 in silico identified EST-SSRs based primers, 44 primers gave cross species amplification and showed their transferability in T. alexandrinum. These identified EST-SSRs may be used in diversity analysis and population genetics studies of germplasm of T. alexandrinum as well as for further breeding programs.

\section{Appendix A. Supplementary data}

Supplementary data associated with this article can be found at http://dx.doi.org/10.2306/ scienceasia1513-1874.2020.001.

\section{REFERENCES}

1. Vilčinskas E, Dabkevičienè G (2009) Qualitative and quantitative characteristics of clover (Trifolium spp.) species in the first year of growing. Zemdirbyste 96, 170-180.

2. Ellison NW, Liston A, Steiner JJ, Williams WM, Taylor NL (2006) Molecular phylogenetics of the clover genus (Trifolium-Leguminosae). Mol Phylogenet Evol 39, 688-705.

3. Treigytè G, Zaikova I, Matuzevičius D, Čeksterytė V, Dabkevičienė G, Kurtinaitienė B, Navakauskienė R (2014) Comparative proteomic analysis of pollen of Trifolium pratense, T. alexandrinum and T. repens. Zemdirbyste 101, 453-460.

4. Badr A, Hanaa H, El-Shazly, Watson LE (2008) Origin and ancestry of Egyptian clover (Trifolium alexandrinum L.) as revealed by AFLP markers. Genet Resour Crop Evol 55, 21-31.

5. Tarrad MM, Zayed EM (2009) Morphological, biochemical and molecular characterization of Egyptian clover (Trifolium alexandrinum L.) varieties. Range Manag Agrofor 30, 115-121.

6. Zehdi S, Trifi M, Billotte N, Marrakchi M, Christophe PJ (2004) Genetic diversity of Tunisian date palms (Phoenix dactylifera L.) revealed by nuclear microsatellite polymprohism. Hereditas 141, 278-287.

7. Malaviya DR, Kumar B, Roy AK, Kaushal P, Tiwari A (2005) Estimation of variability for isozymes of five enzyme systems among wild and cultivated species of Trifolium. Genet Resour Crop Evol 52, 967-976.
8. George J, Sawbridge TI, Cogan NO, Gendall AR, Smith KF, Spangenberg GC, Forster JW (2008) Comparison of genome structure between white clover and Medicago truncatula supports homoeologous group nomenclature based on conserved synteny. Genome 51, 905-911.

9. Malaviya DR, Roy AK, Kaushal P, Kumar B, Tiwari A (2008) Genetic similarity among Trifolium species based on isozyme banding pattern. Plant Systemat Evol 276, 125-136.

10. Squirrell J, Hollingsworth PM, Woodhead M, Russell J, Lowe AJ, Gibby M, Powell W (2003) How much effort is required to isolate nuclear microsatellites from plants?. Mol Ecol 12, 1339-1348.

11. Pashley CH, Ellis JR, McCauley DE, Burke JM (2006) EST databases as a source for molecular markers: lessons from Helianthus. J Hered 97, 381-388.

12. Gupta PK, Rustgi S, Sharma S, Singh R, Kumar N, Balyan HS (2003) Transferable EST-SSR markers for the study of polymorphism and genetic diversity in bread wheat. Mol Genet Genom 270, 315-323.

13. Bhat PR, Krishnakumar V, Hendre PS, Rajendrakumar P, Varshney RK, Aggarwal RK (2005) Identification and characterization of expressed sequence tags-derived simple sequence repeats markers from robusta coffee variety CxR (an interspecific hybrid of Coffea canephora $\times$ Coffea canephora). Mol Ecol Notes 5, 80-83.

14. Shivakumar MS, Ramesh S, Rao AM, Udaykumar HR, Keerthi CM (2017) Cross legume species/genera transferability of SSR markers and their utility in assessing polymorphism among advanced breeding lines in Dolichos bean (Lablab purpureus L.). Int $J$ Curr Microbiol Appl Sci 6, 656-668.

15. Doyle JJ, Doyle JL (1987) A rapid DNA isolation procedure for small quantities of fresh leaf tissue. Phytochem Bull 19, 11-15.

16. Kongkiatngam P, Waterway MJ, Fortin MG, Coulman BE (1995) Genetic variation within and between two cultivars of red clover (Trifolium pratense L.): comparisons of morphological, isozyme, and RAPD markers. Euphytica 84, 237-246.

17. Kölliker R, Jones ES, Jahufer MZZ, Forster JW (2001) Bulked AFLP analysis for the assessment of genetic diversity in white clover (Trifolium repens L.). Euphytica 121, 305-315.

18. Dabkevičienė G, Paplauskienė V, Vilčinskas E (2011) Assessment of genetic diversity in Trifolium spp. using ISSR and RAPD markers. J Food Agr Environ 9, 210-214s.

19. Tangphatsornruang S, Sraphet S, Singh R, Okogbenin E, Fregene M, Triwitayakorn K (2008) Development of polymorphic markers from expressed sequence tags of Manihot esculenta Crantz. Mol Ecol Resour 8, 682-685. 
Appendix A. Supplementary data

Table S1 Identified EST-SSRs and their primers sequences with melting temperatures (TM), length of primers (L) and product size for Trifolium pratense (TP) and Trifolium repens (TR).

\begin{tabular}{|c|c|c|c|c|c|c|c|c|}
\hline Serial & SSRs & Forward primer & $\mathrm{TM}$ & $\mathrm{L}$ & Reverse primer & TM & $\mathrm{L}$ & Size \\
\hline TP1 & (AAG) 5 & CCAACAACAACAAAACAGAGAGAG & 60.231 & 24 & CAACTCACCAAATCAGAAGCAG & 59.914 & 22 & 184 \\
\hline TP2 & (TCG) 4 & AАCTCCACСCAAAАCCACTC & 60.254 & 22 & CAACAACTCATCCACCGACTTA & 60.030 & 22 & 367 \\
\hline TP3 & (CCG) 4 & STTGTGCTGCGCTCTCTCTT & 60.886 & 20 & AACAGCTCTCTGTCCATAAGGC & 59.913 & 22 & 317 \\
\hline TP4 & (CTC) 4 & TCTCCATTCTTCCACCATCTTT & 59.940 & 22 & CTTTCAATGCTTTCATCGTGTC & 59.752 & 22 & 273 \\
\hline TP5 & (CAA) 5 & TCTCCATTCTTCCACCATCTTT & 59.940 & 22 & CTTTCAATGCTTTCATCGTGTC & 59.752 & 22 & 273 \\
\hline TP6 & (TAA)7 & CTGCTTCCACTTTCCTTCAGTT & 59.922 & 22 & GGTGTCTCCTTTGAATACCTGC & 60.004 & 22 & 392 \\
\hline TP7 & (TTC) 4 & CTGCTTCCACTTTCCTTCAGTT & 59.922 & 22 & GGTGTCTCCTTTGAATACCTGC & 60.004 & 22 & 392 \\
\hline TP8 & (TGG) 4 & GAATCGCTTAATTGCTCGTTCT & & 22 & TGCGTTGAAGAATACCATCTTG & 60.131 & 22 & 221 \\
\hline TP9 & (AAAC) 3 & GTCACTTTAGAGGAGCCGGA & 59.430 & 20 & CTTGGAAATGCTTCACCTTTCT & 59.762 & 22 & 386 \\
\hline TP10 & (TGG) 4 & GATACCGGATTTCCATGTCAAC & 50.439 & 22 & CACTTCCAAACTCTTCTTTCGG & & 22 & 395 \\
\hline TP11 & (TTAT) 4 & TACCGGAACCAGAGGGTTA & 9.712 & 21 & ГСАТТССТС & & 22 & 304 \\
\hline TP12 & (TGG) 4 & CAACTAAAGATCAACGGCCAC & 60.413 & 22 & GCAGCTTCCAACCACTGACTAC & 61.237 & 22 & 192 \\
\hline TP13 & GAAA) 3 & GTTGGCTTCCATT & .282 & 22 & ATGATCCA & & 22 & 330 \\
\hline TP14 & (ATTT) 3 & GTCCCAGTTTTGTTGAGAGAGG & 60.146 & 22 & CGTCGGAGAGTGATGATTATTG & 592 & 22 & 298 \\
\hline TP15 & (AAT) 5 & GTCCCAGTTTTGTTGAGAGAGG & 0.146 & 22 & CGTCGGAGAGTGATGATTATTG & & 22 & 298 \\
\hline TP16 & (TGT) 4 & TGTTGAGAGAGG & 60.146 & 22 & CGTCGGAGAGTGATGATTATTG & 59.592 & 22 & 298 \\
\hline TP17 & (TCA) 5 & TTCC & 59.043 & 22 & GTC & & 22 & 223 \\
\hline TP18 & (TGATGG & ACAAATCTGAAGC & 59.757 & 22 & ТССТTСТTСТTСССТ & 60.408 & 22 & 320 \\
\hline TP19 & (TTC)7 & CTC & 60.379 & 22 & CTG & & 22 & 159 \\
\hline TP20 & (ACCT) & GAT & 59.762 & 22 & TGT & 60.461 & 22 & 352 \\
\hline TP21 & (CT)7 & CCI & & 22 & AAG & & 22 & 331 \\
\hline TP22 & (ACC) 1 & GTA & 49 & 21 & ГGGTGCTAATGTTCA & & 22 & 180 \\
\hline TP23 & & & & 22 & & & 22 & 86 \\
\hline TP24 & (ATT & CC & 17 & 22 & TGCT & & 22 & 107 \\
\hline TP25 & & & & 22 & & & 21 & 372 \\
\hline TP26 & TT) 5 & ACG & 07 & 22 & GTC & & 22 & 141 \\
\hline TP27 & & TTGC & & 22 & GTGG & & 22 & 393 \\
\hline TP28 & 4 & CA & .373 & 22 & GTTG & & 22 & 372 \\
\hline TP29 & (AAG) 4 & CC & & 22 & & & 22 & 265 \\
\hline TP30 & (GT) & TTA & .089 & 22 & AGG & & 22 & 190 \\
\hline TP31 & (TTA & $\mathrm{AG}$ & & 22 & ГTAG & & 22 & 341 \\
\hline TP32 & 5 & GCA & 715 & 22 & CCT & & 22 & 229 \\
\hline TP33 & $(1$ & GACAAG & & 25 & AAG & & 22 & 199 \\
\hline TP34 & ( & & 99 & 22 & GA & & 22 & 283 \\
\hline TP35 & & CT & & 22 & & & 22 & 364 \\
\hline TP36 & & $\mathrm{CT}$ & & 22 & & & 21 & 369 \\
\hline TP37 & 10 & & & 22 & & & 22 & 292 \\
\hline TP38 & & $\mathrm{AC}$ & & 22 & & & 22 & 194 \\
\hline TP39 & (') & CTC & & 22 & STGA & 52 & 22 & 147 \\
\hline TP40 & & & & 2 & & & 22 & 147 \\
\hline TP41 & & & 82 & 22 & & & 22 & 330 \\
\hline & & & & 2 & & & 2 & 362 \\
\hline TP43 & & & & 2 & & & 22 & 288 \\
\hline & & & & 2 & & & & 20 \\
\hline TP45 & & & & 22 & GA & & 22 & 196 \\
\hline & & & & 2 & TGC & & & 84 \\
\hline TP47 & & & & 2 & & & 22 & 331 \\
\hline & & & & 2 & & & 2 & 43 \\
\hline TP49 & & AC & & 2 & & & 22 & 36 \\
\hline TP50 & & & & 2 & & & & \\
\hline TP51 & & CTT & & 2 & & & 22 & 59 \\
\hline 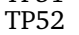 & & & & ? & & & & 89 \\
\hline TP53 & & & & 2 & & & 22 & 370 \\
\hline & & & & 2 & & & & 218 \\
\hline TP55 & & & & 20 & AG & & 22 & 193 \\
\hline & & & & & & & & 324 \\
\hline TP57 & & & & 2 & & & 22 & 141 \\
\hline D & & 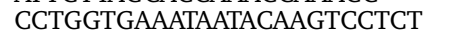 & & 2 & & & 22 & 259 \\
\hline TP & & & & 1 & & & 22 & 375 \\
\hline & & & & & & & 22 & 368 \\
\hline TP61 & & & & 2 & & & 22 & 346 \\
\hline & & & & 22 & & & 22 & 20 \\
\hline TP & & & & 22 & & & 22 & \\
\hline TP64 & & & & 22 & & & 22 & 357 \\
\hline 1 & & & & 2 & AGT & & 22 & 219 \\
\hline & & & & 2 & & & 22 & \\
\hline 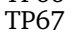 & & & & 2 & & & 22 & OU \\
\hline TP68 & & & & 2 & GA & & 22 & \\
\hline & & & & 2 & & & 23 & 378 \\
\hline & & & & 22 & & 323 & 23 & \\
\hline
\end{tabular}


Table S1 Continue.

\begin{tabular}{|c|c|c|c|c|c|c|c|c|}
\hline Serial & SSRs & Forward primer & TM & $\mathrm{L}$ & Reverse primer & TM & $\mathrm{L}$ & Size \\
\hline$? 71$ & 9 & A & 59.437 & 22 & A & 9 & 22 & 284 \\
\hline TP72 & (TCT) 4 & GTTTCCGCTACCACATCTTTTC & 60.007 & 22 & GTTTCCAACTCTCTTCACACCC & 60.016 & 22 & 162 \\
\hline TP73 & (TGT) 4 & GTTTCCGCTACCACATCTTTTC & 60.007 & 22 & TCTTCTGTTCTAACCAGCCCTC & 59.886 & 22 & 314 \\
\hline TP74 & (TCA) 4 & AAACGATAGTGTGGATTTTGCC & & 22 & ATGAAGATTTCCGTGTCATTCC & & 22 & 129 \\
\hline TP75 & (GTA) 4 & AACACTTCACCGGACACTCTTT & 60.074 & 22 & TCTTTCCTATCCACAACTGGCT & 60.130 & 22 & 297 \\
\hline TP76 & (CAAAC) 4 & ССТСТTСТСАТСТСТССТTCСА & 59.950 & 22 & CAATGATGGGTTGGTGCTAAA & 60.737 & 21 & 184 \\
\hline TP77 & (ATG) 4 & CTTAACTCAAATGACTCGCCTT & 57.715 & 22 & GAAACATCTTCCСTTTCAACAC & 57.695 & 22 & 309 \\
\hline TR1 & (CAT) 4 & TGATGGAATGGATGAAGATGAG & 59.894 & 22 & AGTGATTCGGCAACCTTCTCTA & & 22 & 333 \\
\hline TR2 & (CAC) 4 & GATCCATCTCCСАCACTTAAACA & 60.235 & 23 & TTGGTGTTGTGCTTCTGGACTA & 60.721 & 22 & 199 \\
\hline TR3 & (ACC) 5 & СССАAАACСТАAАССТСАAАAC & 58.948 & 22 & TGCTCTGTACCTTTCTTGACCA & 59.914 & 22 & 386 \\
\hline TR4 & (ATT) 6 & TTAACCACCACCACCACCTTAT & 60.385 & 22 & TGCTCTGTACCTTTCTTGACCA & 59.914 & 22 & 333 \\
\hline TR5 & (CAA) 4 & TGCTCAAATTCAAGTATGCCAC & 60.138 & 22 & CAAAGTCACCTCACATCTACGC & 59.803 & 22 & 281 \\
\hline TR6 & (GAA) 4 & GCGTAGATGTGAGGTGACTTTG & 59.803 & 22 & TAAGGGAGGCATGTCGAAGTAT & 59.987 & 22 & 313 \\
\hline TR7 & (TCGC) 3 & CACTAACATTCACTTGCATACACAC & & 25 & GATTCTTCGTCCAGGCTATTGT & & 22 & 232 \\
\hline TR8 & (GATTTT) & TCCGATTTCACAACCTTCCTTA & 60.821 & 22 & ATTCAAAGACGGGAATCTGTGT & 59.870 & 22 & 164 \\
\hline TR9 & (TGTT) 3 & TTCTGATTTCACACCGTTTCAG & & 22 & ATAACGACCCTTTCGGGTTAAT & & 22 & 368 \\
\hline TR10 & (TCA) 5 & ACTACTACTGATGGCGTGTCTCC & 59.718 & 23 & GCGATGTTGTTGTTGATAGGAA & 60.003 & 22 & 109 \\
\hline TR11 & (TTCC) 3 & АTTTCССТСТАAССТGCATCTG & 59.615 & 22 & GATACTTGCTACCGGAATCGAC & 59.993 & 22 & 309 \\
\hline TR12 & (TTTC) 3 & ATTTCCCTCTAACCTGCATCTG & 59.615 & 22 & GATACTTGCTACCGGAATCGAC & 59.993 & 22 & 309 \\
\hline TR13 & (CAG) 4 & CСATCCTTATTCACTTT & 59.898 & 22 & GTCTTCGGTCTACTCC & 59.998 & 22 & 388 \\
\hline TR14 & (CAC) 4 & GAAGCTATGGAATCAAGGAAGC & 59.359 & 22 & TCAACAAACAAACCAAGAGTGG & 60.045 & 22 & 361 \\
\hline TR15 & (CAC) 4 & GAAGCTATGGAATCAAGGAAGC & 59.359 & 22 & TCAACAAACAAACCAAGAGTGG & 60.045 & 22 & 361 \\
\hline TR16 & (TTTG) 3 & TATTTCCACCGAAGAATCAAGG & 60.305 & 22 & TCAAGCCAAACTTCCT & & 22 & 107 \\
\hline TR17 & (GAA) 4 & GCGTAGATGTGAGGTGACTTTG & 59.803 & 22 & TAAGGGAGGCATGTCGAAGTAT & 59.987 & 22 & 313 \\
\hline TR18 & (ATC) 5 & СССАТСАССТ & 59.901 & 22 & GTATTTCCTGCI & 60.270 & 22 & 324 \\
\hline TR19 & (CAC)7 & GAAATGTGTTCTTGGCGTTGTA & 60.039 & 22 & ГTAACAATCCATC & 59.970 & 22 & 194 \\
\hline TR20 & (ACT) 4 & [GGCTATGAAACGTG & 60.131 & 22 & AGGC & 59.968 & 22 & 115 \\
\hline TR21 & (GCT) 4 & ГСССАAТ & 60.178 & 22 & GTAACCAGGCAC & 59.877 & 22 & 385 \\
\hline TR22 & (ССATCA) 3 & CTC & & 20 & G & & 21 & 376 \\
\hline TR23 & (CT) 14 & СТСТСАТСССТСТТССАСТСТС & 59.439 & 22 & GGGAGTTCGAGTTCAGTTATGG & 59.998 & 22 & 109 \\
\hline TR24 & (TCT) 6 & TCC & & 20 & & & 22 & 283 \\
\hline TR25 & (TTCC) 3 & TTGAAGATGGCTATGAAACGTG & 60.131 & 22 & AGGCATGAAGAT" & 59.968 & 22 & 115 \\
\hline TR26 & (GCT)4 & ГCATCGAATCCCAAT & 60.178 & 22 & CCTC & 59.877 & 22 & 385 \\
\hline TR27 & (ACC) 4 & GGT & 60.036 & 22 & & 60.247 & 22 & 198 \\
\hline TR28 & & TC & & 22 & GT & & 22 & 364 \\
\hline TR29 & (TCTT) 3 & TCC & 60.019 & 22 & AGT & 60.131 & 22 & 296 \\
\hline TR30 & (CAA) 4 & ACCC & & 22 & & & 22 & 389 \\
\hline TR31 & (GCC) 4 & ICCTG & 59.954 & 24 & AAT & 59.968 & 22 & 115 \\
\hline TR32 & (ATTC) 4 & GGAA & 60. & 23 & GGT & 12 & 22 & 398 \\
\hline TR33 & (CCT) 4 & AGGC & 59.928 & 22 & AGC & 59.997 & 20 & 267 \\
\hline TR34 & & & & 22 & & & 22 & 281 \\
\hline TR35 & (GAA) 4 & TTG & 59.803 & 22 & GTAT & 59.987 & 22 & 313 \\
\hline TR36 & (ATC) 8 & ACCCA & 59 & 22 & $\mathrm{TC}$ & 27 & 22 & 395 \\
\hline TR37 & (TTC) 8 & TCC & 60.075 & 22 & TGTT & 30 & 22 & 233 \\
\hline TR38 & (ACA) 6 & ССТTТСТC & 60.019 & 22 & CTT & 38 & 22 & 272 \\
\hline TR39 & G) 4 & CGTGC & 60.401 & 22 & CTGAGAA & 59.633 & 22 & 229 \\
\hline TR40 & & & 63.228 & 22 & & & 18 & 105 \\
\hline TR41 & (1 & & & 22 & A & & 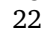 & 329 \\
\hline TR42 & (TTCTCT) 3 & CT & 1 & 22 & GGA & & 22 & 329 \\
\hline TR43 & & & & 22 & AGA & & 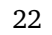 & 115 \\
\hline TR44 & & & & 22 & CAT & & 23 & 188 \\
\hline TR45 & & TG & & 22 & TAT & & 2 & 313 \\
\hline TR46 & & & & 22 & & & 22 & 296 \\
\hline TR47 & & CTC & & 25 & & & 22 & 390 \\
\hline TR48 & Г) 4 & G & 60 & 20 & CC & 08 & 21 & 243 \\
\hline TR49 & & & & 22 & & & & 358 \\
\hline TR50 & & & & 22 & TTA & & 22 & 379 \\
\hline TR51 & & & & 23 & $\mathrm{AG}$ & & 22 & 234 \\
\hline TR52 & (AAT) 5 & CG & & 22 & CAC & & 22 & 400 \\
\hline TR53 & (TTTGAT) 4 & & 59.971 & 22 & TTTC & & 22 & 376 \\
\hline TR54 & (CTTC) 3 & ATCACAACACACACC & 58.959 & 22 & CCATCAGTTTAT & 59.775 & 22 & 209 \\
\hline TR55 & & & & 22 & & & 22 & 350 \\
\hline TR56 & A) 7 & SATAC & 4 & 22 & GTC & & 22 & 198 \\
\hline TR57 & & & & 22 & & 75 & 22 & 170 \\
\hline TR58 & & AGC & & 22 & & & 22 & 290 \\
\hline TR59 & & CAGC & & 22 & CCT & & 22 & 290 \\
\hline TR60 & (AAC) 4 & CAGCTCTGATTCTTT & & 22 & САTCTAATCСТTCT & & 24 & 136 \\
\hline TR61 & & & & 22 & CAACCTTTGGTG & & 22 & 306 \\
\hline TR62 & & ATTTCCTTATCTTCTT & & 22 & GTCCTAAAAGCAACCTTTGGTG & & 22 & 306 \\
\hline TR63 & & CTACCACCACCGTA & & 21 & CATGGTTCTTCTTATCTCCTCTGA & 34 & 25 & 196 \\
\hline TR64 & & 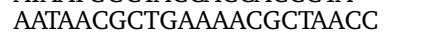 & & 22 & TGAA & & 22 & 137 \\
\hline TR65 & & AGC & & 22 & TGA & & 22 & 372 \\
\hline TR66 & & ACAGA & & 22 & ACAAAGCAACAACCCCAATTAC & & 22 & 378 \\
\hline TR67 & & & 60. & 2 & AGATGCTGA & & 22 & 183 \\
\hline TR68 & (GAA) 6 & TGTTGAAGATAGCGACGAAGAA & 60.018 & 22 & TCACACTCACCATGACAAATCA & 60.008 & 22 & 387 \\
\hline
\end{tabular}


Table S1 Continue.

\begin{tabular}{|c|c|c|c|c|c|c|c|c|}
\hline Serial & SSRs & Forward primer & $\mathrm{TM}$ & $\mathrm{L}$ & Reverse primer & $\mathrm{TM}$ & $\mathrm{L}$ & Size \\
\hline TR69 & 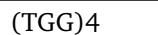 & CCAAAGAGA & 61.083 & 22 & GACC & 60.357 & 22 & 2 \\
\hline TR70 & (TTCA) 3 & GTTTCACCACAACAACAACAC & 59.410 & 22 & CCTTGTCCTGTTCGTCTTCTCT & & 22 & 257 \\
\hline TR71 & (TC) 8 & TGTTTCACCACAACAACAACAC & 59.410 & 22 & CCTTGTCCTGTTCGTCTTCTCT & 59.916 & 22 & 257 \\
\hline TR72 & (TGTT) 3 & TTCTGATTTCACACCGTTTCAG & 60.147 & 22 & ATAACGACCCTTTCGGGTTAAT & 59.975 & 22 & 368 \\
\hline TR73 & (ACCTCC) & AATCTGCTGGAATGATGGTTG & 59.947 & 21 & GAGGTGATTGTGATGACTGAGC & 59.734 & 22 & 141 \\
\hline TR74 & (AGG) 4 & AACACCAGAAACTGAACGACCT & 60.074 & 22 & GGCATTTGACCATAAACAACAG & 59.384 & 22 & 264 \\
\hline TR75 & (AT) 8 & AAGATGGAGCATTTCCGAGTAG & 59.747 & 22 & CTAATTCAAGGGGTTTTGCTTG & 59.997 & 22 & 375 \\
\hline TR76 & (TCT) 4 & CACGAGGTTCTCTTCTTCCATT & 59.751 & 22 & CCTTTAGCAGCAGCAGATTGA & & 21 & 373 \\
\hline TR77 & (TTAT) 6 & TCTCTTCTTCСATTCССТCTTC & 58.920 & 22 & GTGATAAGCATGAACCCAGTTG & 59.499 & 22 & 252 \\
\hline TR78 & (TCTA) 3 & TCTСТTСТСТТСТСТTСТСТАTCAAGG & 59.416 & 27 & CСACСACCACCATTCATATTC & 59.925 & 21 & 210 \\
\hline TR79 & (TGG) 4 & TCTATATGCAGCTTGGTGATGC & 60.259 & 22 & TCCTTCTGATTTTCCACTTCTA & 60.005 & 24 & 389 \\
\hline TR80 & (CAAT) 3 & CTTTCAGAGACACTTCGCTTCA & 59.797 & 22 & GAAGAACTCATCTCGCTCCAGT & 60.023 & 22 & 149 \\
\hline TR81 & (ATT) 4 & TAGCTCAGAAATGCAAAAGTGG & 59.536 & 22 & GTTGATACTCGTCCACAACGAC & 59.525 & 22 & 362 \\
\hline TR82 & (AGA) 5 & TATTGCTCTGCGAAGGGTTATT & 60.115 & 22 & ATGATCGGAAGACCTGATAGGA & 59.925 & 22 & 195 \\
\hline TR83 & (ACC) 4 & GGTTGTGCATCTTCTAAACGTG & 59.677 & 22 & TGTGGTTGTGATTGTTGTGATG & 60.324 & 22 & 320 \\
\hline TR84 & (CCAAAC) 4 & CGTCTCACTTGTTCTTCCCTCT & 59.916 & 22 & GAATCTCCTTGCTGCCTTAGAA & 59.987 & 22 & 345 \\
\hline TR85 & (CCAAAC) 4 & TTCAAGGACCACTACCAATGC & 59.985 & 21 & AGCGAA & & 22 & 341 \\
\hline TR86 & (ATC) 5 & ACACCACCATCATA & 59.724 & 22 & AGACCGAAAGGGAGATAGAACC & 59.968 & 22 & 218 \\
\hline TR87 & (TTCC) 3 & AAAGAGAT & 60.261 & 21 & А ССА АТТА САТ & 396 & 22 & 233 \\
\hline TR88 & (CTT) 10 & CCAAATC & 60.008 & 22 & GCGGT & 9.913 & 22 & 345 \\
\hline TR89 & (CCA) 5 & CAAG & 60.037 & 22 & GCTA & 60.096 & 22 & 200 \\
\hline TR90 & (CTTC) 3 & TCACAATCACAACACACACCC & 60.326 & 21 & TTTAT & 59.775 & 22 & 220 \\
\hline TR91 & (AATA) 4 & TCCT & 59.833 & 22 & TGC & 102 & 22 & 363 \\
\hline TR92 & (TGC) 4 & CCA & 60.008 & 22 & $\mathrm{CC}$ & 60.042 & 22 & 400 \\
\hline TR93 & (ATTA) 3 & GTG & 60.291 & 22 & TTA & 92 & 22 & 386 \\
\hline TR94 & (GGT) 4 & $\mathrm{~T}$ & 59.225 & 20 & ГАА & 58.766 & 22 & 292 \\
\hline TR95 & (AATC) 3 & AGC & & 22 & GTC & 525 & 22 & 170 \\
\hline TR96 & (CAC) 4 & $\mathrm{AC}$ & 60.208 & 21 & TTA & 59.747 & 22 & 366 \\
\hline TR97 & ACC) 3 & AA & & 22 & CT & & 22 & 266 \\
\hline TR98 & (ACA) 4 & TGTA & 59.998 & 22 & TCT & 950 & 22 & 276 \\
\hline 9 & $(1)+2)$ & & & 21 & $\mathrm{BAC}$ & 43 & 22 & 198 \\
\hline TR100 & (GGT) 4 & CTGAA & 60.134 & 25 & $\mathrm{TA}$ & 59.238 & 21 & 196 \\
\hline TR101 & (c) & & & 22 & & & 22 & 373 \\
\hline TR102 & (ATC) 4 & TTT & 60.364 & 22 & TTT & 60.166 & 21 & 249 \\
\hline & $(\mathrm{C}$ & & & 22 & & & 22 & 314 \\
\hline TR104 & (GA & & 59 & 22 & & 60.127 & 22 & 175 \\
\hline TP105 & (G & & & 22 & & & 22 & 168 \\
\hline TR106 & (AAC) 8 & AT & 60.262 & 22 & GC & 60.315 & 22 & 137 \\
\hline TD 107 & (CG & & & 22 & & & 21 & 379 \\
\hline TR108 & (ATCA) 4 & $\mathrm{TC}$ & 59.969 & 22 & CT & 59 & 22 & 383 \\
\hline TD100 & (GAA) 4 & & & 22 & & & 22 & 349 \\
\hline TR110 & $\left(\mathrm{GA}^{\prime}\right.$ & $\mathrm{GC}$ & 60.136 & 22 & & 59.987 & 22 & 219 \\
\hline TR111 & (TC & & & 21 & AA & & & 356 \\
\hline TR112 & (ACTA) 3 & [TATGGTTTAGTTG & 60.739 & 22 & AATCCCAGCATGTTTGTTATCC & 60.082 & 22 & 358 \\
\hline TR113 & & & & 22 & & 59.920 & 22 & 301 \\
\hline & (AG & GAAAAGGAAATAA & 60.3 & 22 & TTGGCGAGTGTCGTA & & 22 & 230 \\
\hline TR115 & (CCG) 4 & & 59.899 & 21 & IGTTG & 59.780 & 22 & 264 \\
\hline & (ATC) 4 & GTCTCATC & & 22 & ACGAGGTGATTCI & & 22 & 218 \\
\hline & (CGC) 4 & & 59.499 & 22 & GGAGTTCCGTTA & 60.004 & 22 & 174 \\
\hline TR118 & (TGC) 5 & AATAGGTGGTGGATTGGATCTG & 60.074 & 22 & CCCCTTGATTACTCCTACCTGA & 59.477 & 22 & 269 \\
\hline TR119 & (AGTG) 4 & CGG & & 19 & GACTCGTTGT & 59.668 & 22 & 162 \\
\hline TR120 & (CAA) 4 & GCC & & 21 & CCA & & 22 & 287 \\
\hline TR121 & (TGG) 5 & ACTGAGCTTTGTCTTGGTTTGC & 60.831 & 22 & CTACTTGAGCCTTAGCTGGTGG & 60.436 & 22 & 226 \\
\hline
\end{tabular}

